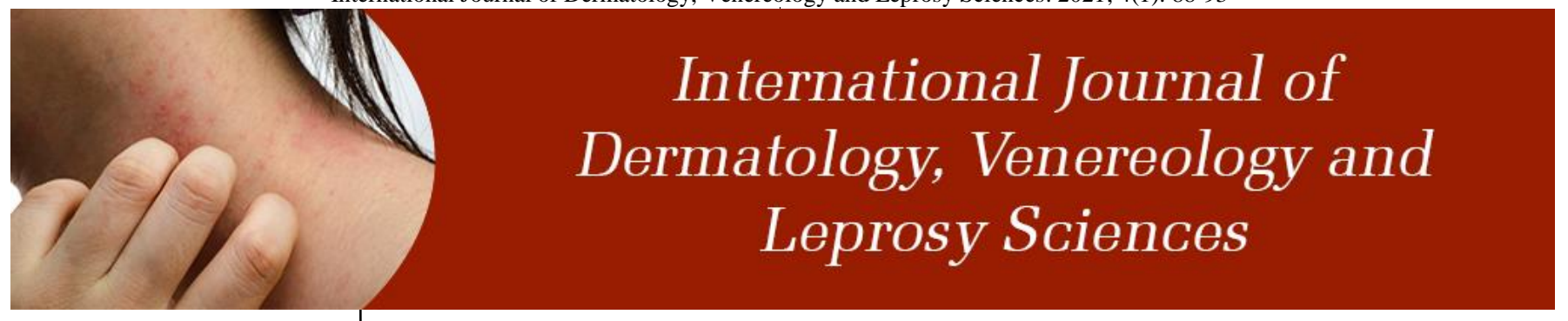

E-ISSN: 2664-942X

P-ISSN: 2664-9411

www.dermatologypaper.com

Derma 2021; 4(1): 88-93

Received: 15-11-2020

Accepted: 21-12-2020

Yurike Indah Pratiwi

General Practitioner, Faculty of Medicine, Tarumanagara

University, Jakarta, Indonesia

Cindy Christella Chandra General Practitioner, Faculty of Medicine, Tarumanagara University, Jakarta, Indonesia

Sukmawati Tansil Tan Department of DermatoVenereology, Faculty of Medicine, Tarumanagara University, Jakarta, Indonesia

Corresponding Author: Yurike Indah Pratiwi General Practitioner, Faculty of Medicine, Tarumanagara University, Jakarta, Indonesia

\section{A case report: Secretome of Wharton's jelly-derived MSCS (SWJ-MSCS) for treatment of Echtyma Gangrenosum}

\author{
Yurike Indah Pratiwi, Cindy Christella Chandra and Sukmawati Tansil \\ Tan
}

DOI: https://doi.org/10.33545/26649411.2021.v4.i1b.73

\begin{abstract}
Polythene is the most common plastic. The annual global production is around 80 million tones. It's Ecthyma gangrenosum (EG) is an uncommon skin infection caused most commonly by Pseudomonas aeruginosa. It is characterized by a macule that rapidly changes into a gangrenous ulcer with a black eschar surrounded by erythema. We report a case of EG in a previously healthy woman treated with intracutaneous injection and topical gel of Wharton's Jelly-derived MSCs (SWj-MSCs) secretome that showed a significant result in the wound healing process, and the patient was satisfied with the results.
\end{abstract}

Keywords: Echtyma gangrenosum, secretome, stem cell, wharton's jelly, wound healing

\section{Introduction}

Ecthyma gangrenosum (EG) is a skin infection caused by microorganisms such as bacteria (Pseudomonas aeruginosa, Staphylococcus aureus, Group A Streptococcus, Escherichia coli, Klebsiella pneumoniae, Serratia marcescens and Aeromonas hydrophila) and fungi (Candida albicans) but most commonly by Pseudomonas aeruginosa ${ }^{[1,2]}$. EG usually targets immunocompromised subject's body parts such as on the anogenital area, buttocks, extremities, abdomen, axillae, and rarely on the face. Despite this, it has the potential to affect previously healthy subjects ${ }^{[3]}$. The mortality rate varies between $35 \%$ and $90 \%$, depending on the severity of the underlying sepsis, which can be as low as $15 \%$ in subjects without bacteremia. Septic shock, neutropenia, resistant microorganisms, and inadequate or delayed antibiotic treatment are all associated with increased mortality ${ }^{[2,4]}$. Antibiotics such as third and fourth-generation cephalosporins, broad-spectrum penicillins, $\beta$-lactam antibiotics, and aminoglycocides can be used for EG treatment. Surgical debridement may be necessary to prevent further spread of infection for necrotic tissue and drainage of localized abscesses $[1,2,5]$. The secretome is a product secreted by MSCs that contains a variety of growth factors, cytokines, and chemokines that aid in the re-epithelialization, immunomodulation, angiogenesis, and ECM remodeling processes, thereby accelerating wound healing. This case report discusses the potential benefit of treating ecthyma gangrenosum with secretome. The secretome is used in conjunction with other therapies to support in wound healing ${ }^{[6]}$.

\section{Case Report}

A 37-year-old woman presented with a 3-day history of a black wound on her left thigh near her groin. Initially, it looked like a red papule that was neither itchy nor painful. In 24 hours, this red papule turned blackish and increased in size. The patient had no history of immune system disorders and was not taking immunosuppressive drugs. On examination, the patient was hemodynamically stable, and there was no fever. On the dermatological examination on the left thigh, a necrotic ulcer covered with black eschar, measuring $17 \times 7 \mathrm{~cm}$, with a hyperemic edge, was found (fig.1). The patient was initially given intravenous thirdgeneration cephalosporin for broad-spectrum coverage. The lesion was cleaned with $\mathrm{NaCl}$, followed by applying topical antibiotics. Twelve days later, the black eschar became hard, and the erythema became less (fig. 2A). The patient consented to surgical debridement. 
After that, the secretome of Wharton's Jelly-derived MSCs (SWj-MSCs) was injected intracutaneously of the lesion (fig 2B). The patient also was given $\mathrm{SWj-MSCs}$ gel to be applied daily by herself at home. After two days, the patient came back to control; tissue regeneration and repair process had occurred. The lesion looked clean as the vessel contracted and the leaked blood coagulated (fig. 3). Sixteen days after the intervention, the formation of granulation tissue has appeared (fig. 4). Two weeks later, the lesion has got smaller in size, and granulation tissue was perfectly formed (fig. 5). The lesion had been getting better since then (fig.6), and one month later, it became a closed wound accompanied by scarring (fig. 7). The patient was very satisfied with the result.

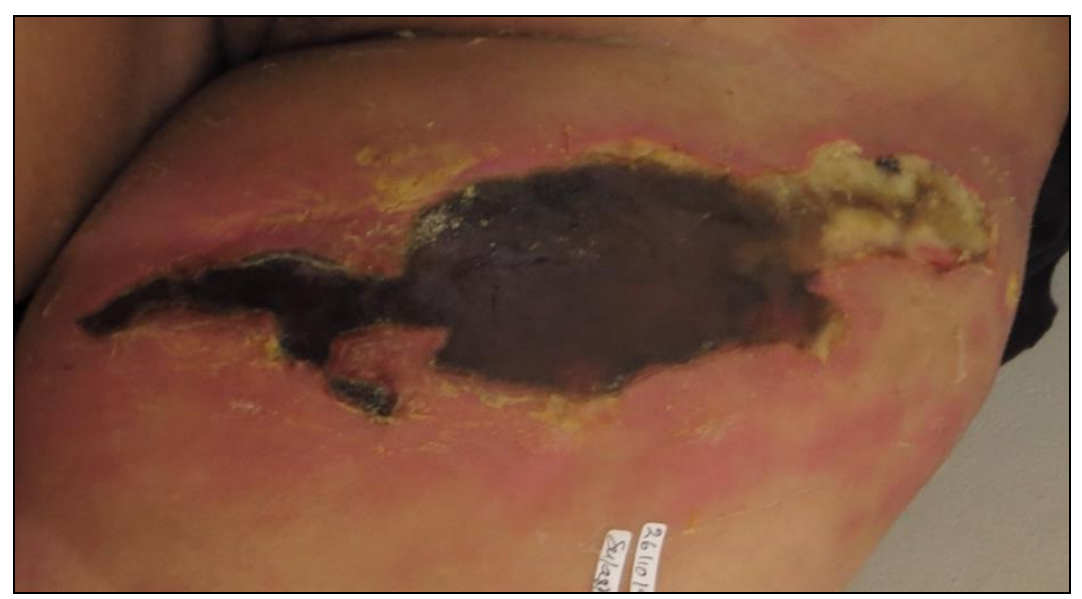

Fig 1: A necrotic ulcer covered with black eschar, measuring $17 \times 7 \mathrm{~cm}$, with a hyperemic edge

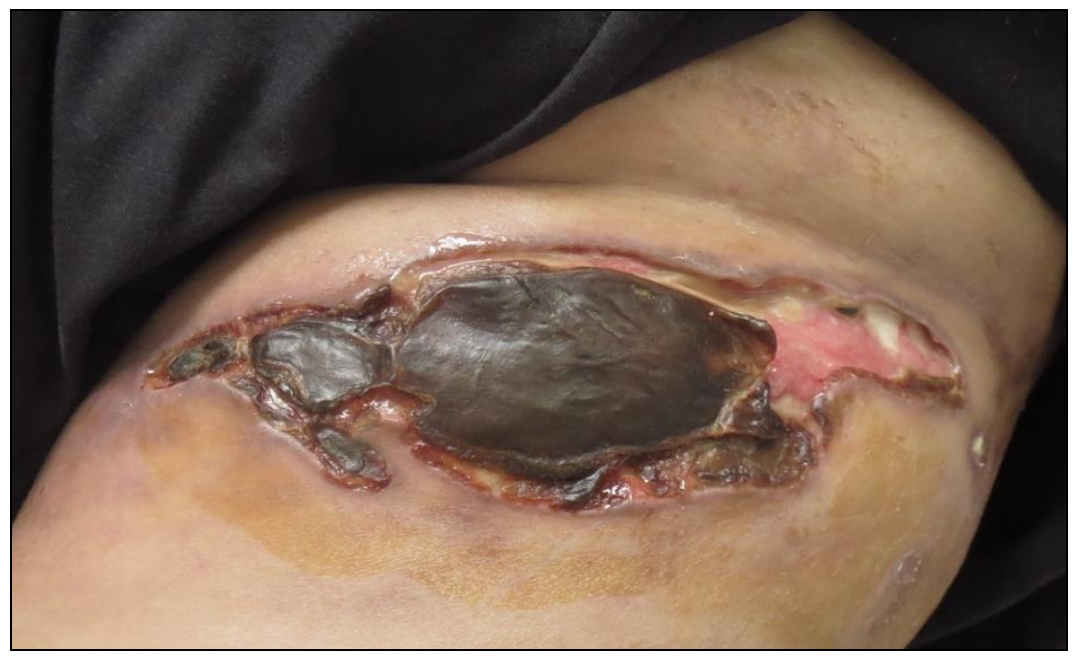

Fig 2A: Day 12. Before Debridement

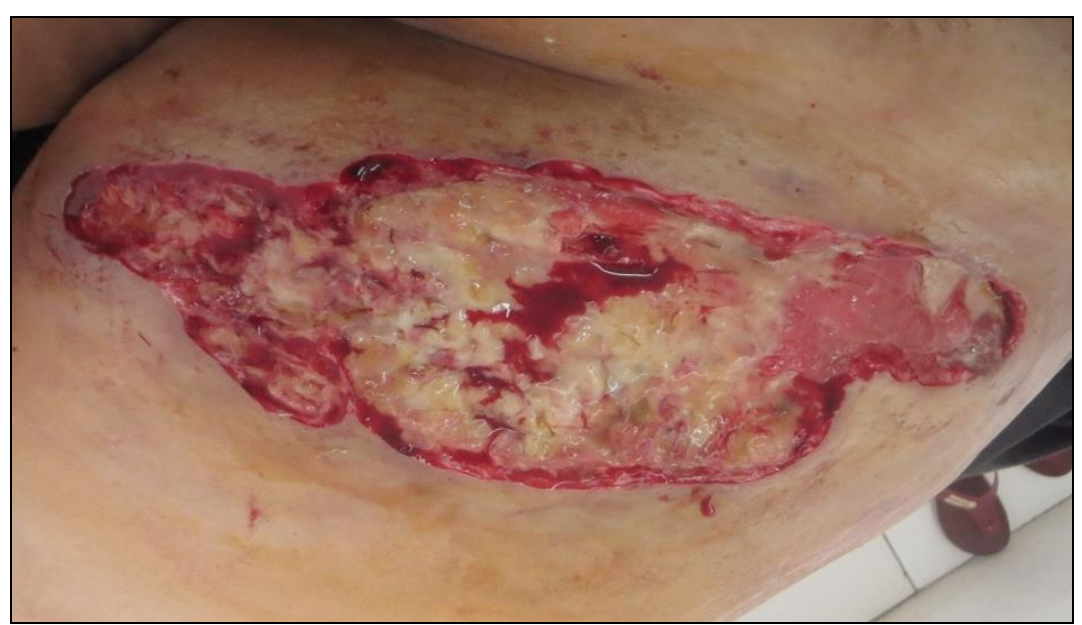

Fig 2B: After debridement, the wound was injected intracutaneously with secretome of Wharton's Jelly-derived MSCs (SWj-MSCs) 


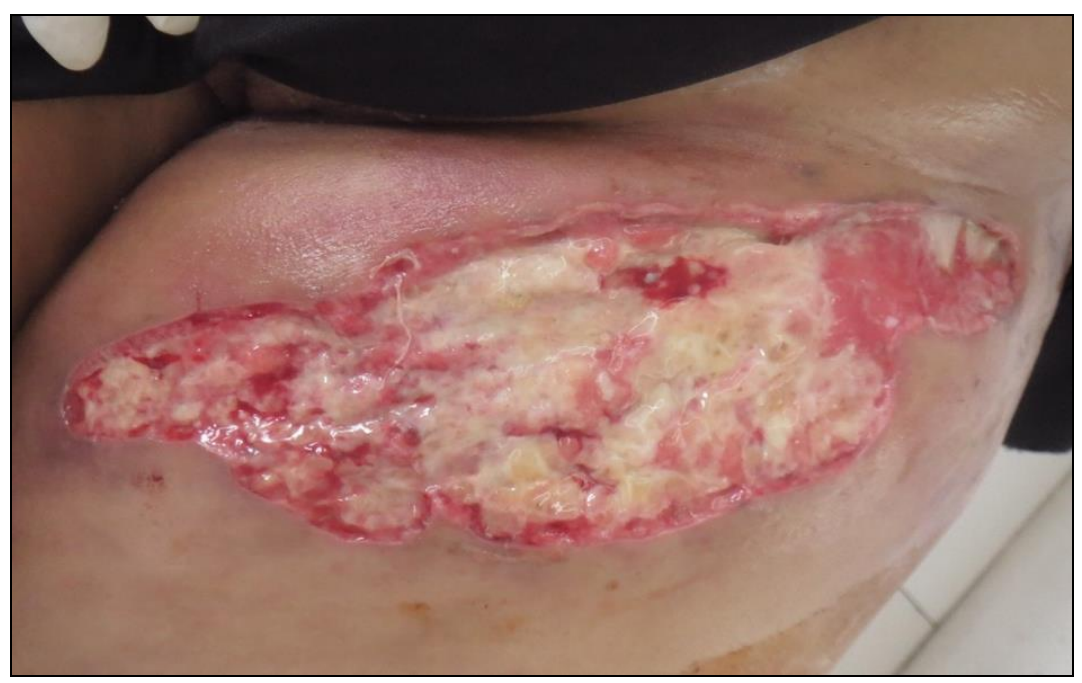

Fig 3: Two days after intervention: The lesion looked clean as the vessel contracted and the leaked blood coagulated.

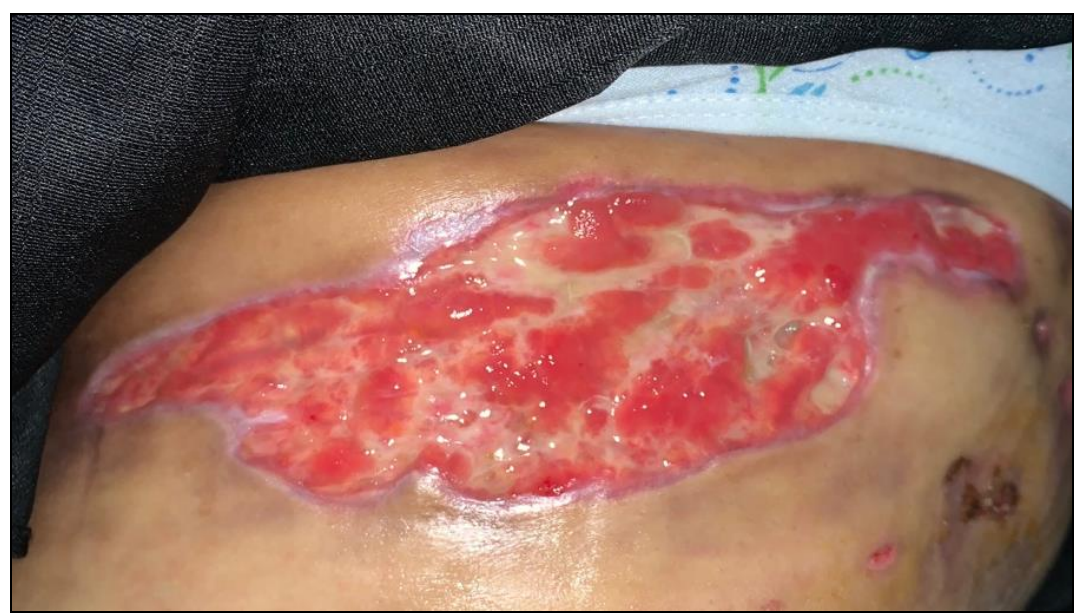

Fig 4: Sixteen days after the interventions: Formation of granulation tissue has appeared.

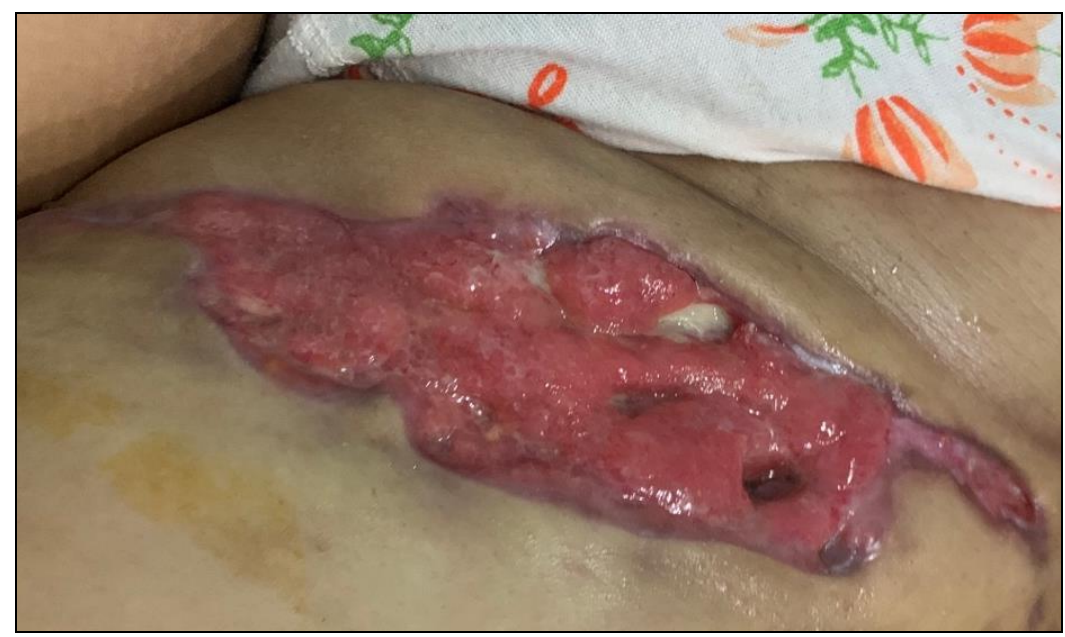

Fig 5: Thirty days after the intervention: The lesion has got smaller in size, and granulation tissue was perfectly formed. 


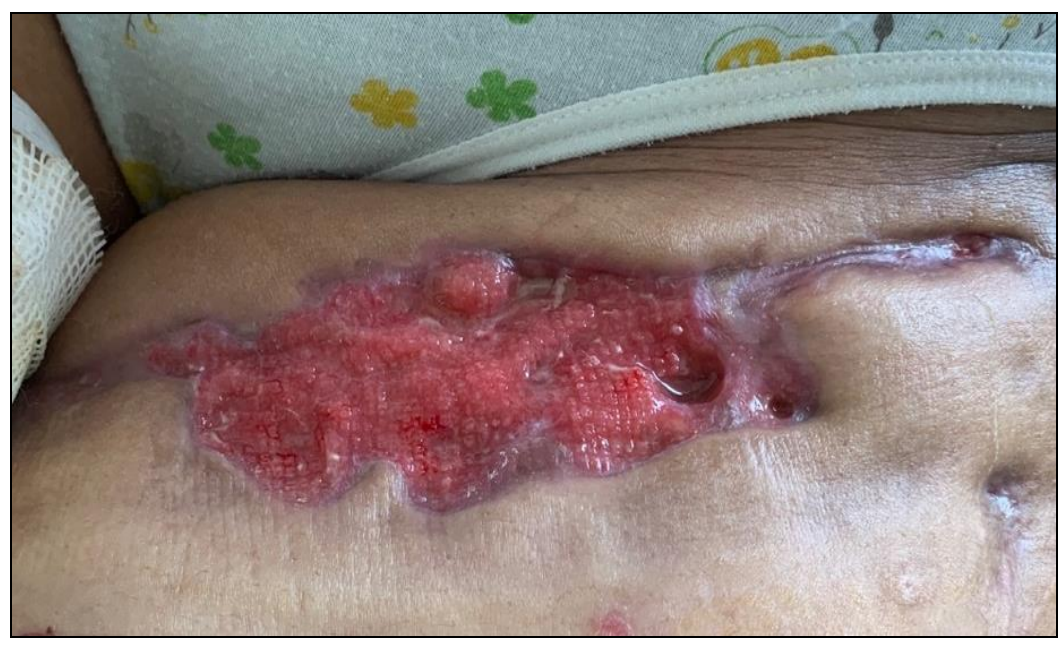

Fig 6: Fifty days after the intervention

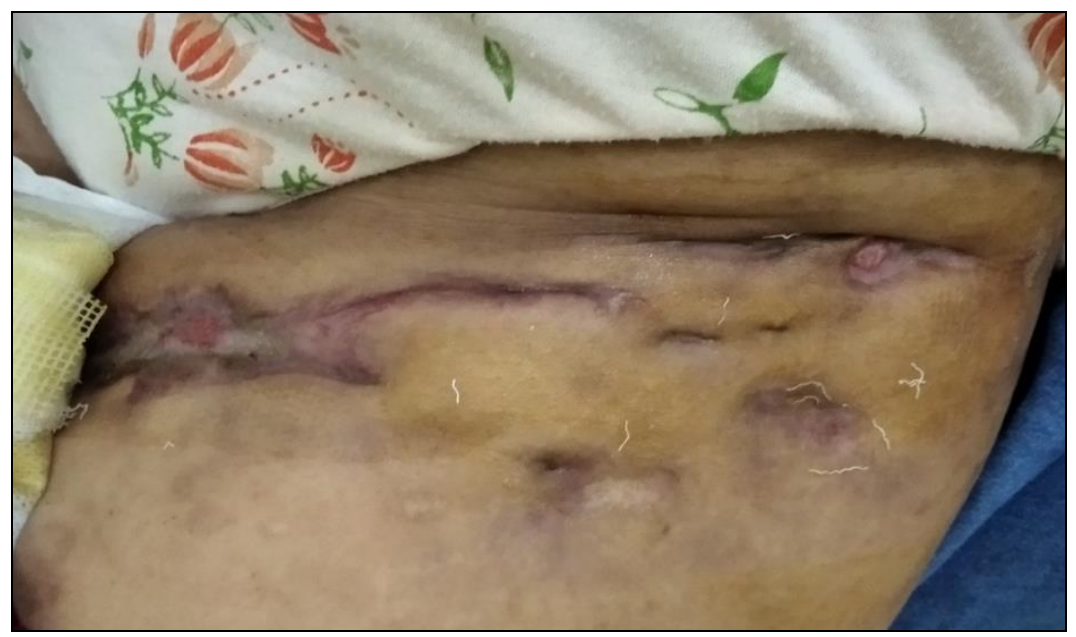

Fig 7: One month later, the patient came to control. The wound has closed and was accompanied by scarring

\section{Discussion}

Ecthyma gangrenosum (EG) is a rare type of skin infection that is most frequently caused by Pseudomonas aeruginosa. EG manifests clinically as a painless, erythematous macule that progresses to form a papule, pustule, nodule, or bulla. It develops to a gangrenous ulcer with a black eschar surrounded by erythema within 12 hours ${ }^{[7]}$. There have been two distinct forms of EG described. Bacteria spread hematogenously and infect the media and adventitia of blood vessels in the classic bacteremic form. Ischemic necrosis of the surrounding skin occurs as a result of perivascular invasion. In the second nonbacteremic form, the lesions occur at the site of direct inoculation into the skin. Pseudomonas aeruginosa produces protease, elastase, phospholipase $\mathrm{C}$, and exotoxin A that are responsible for the direct tissue and vessel destruction leading to ulcerative lesions ${ }^{[5,8,9]}$.

Mesenchymal Stem Cells (MSCs) are one type of stem cells that can differentiate into a variety of tissue-specific cell types. Due to the proliferative and regenerative capabilities of stem cells, they are currently an effective alternative therapy for skin diseases. MSCs can be isolated from a variety of tissue types, including bone marrow, adipose tissue, skeletal muscle, dental pulp, amniotic membrane, amniotic fluid, and umbilical cord ${ }^{[10,11]}$.

MSCs contribute to the cutaneous wound healing process by expediting wound closure, accelerating re-epithelialization, improving tissue granulation, inducing angiogenesis, reducing inflammation, and increasing ECM remodeling. These beneficial effects are apparently mediated by paracrine signaling. Certain cells, such as epithelial cells, endothelial cells, keratinocytes, and fibroblasts, respond to MSC paracrine signaling. MSC paracrine signaling controls a variety of cellular responses to environmental changes, including cell survival, proliferation, migration, and gene expression, via the secretion of bioactive molecules such as cytokines, chemokines, and growth factors, collectively referred to as secretome. (Fig. 8) ${ }^{[12-14]}$. 


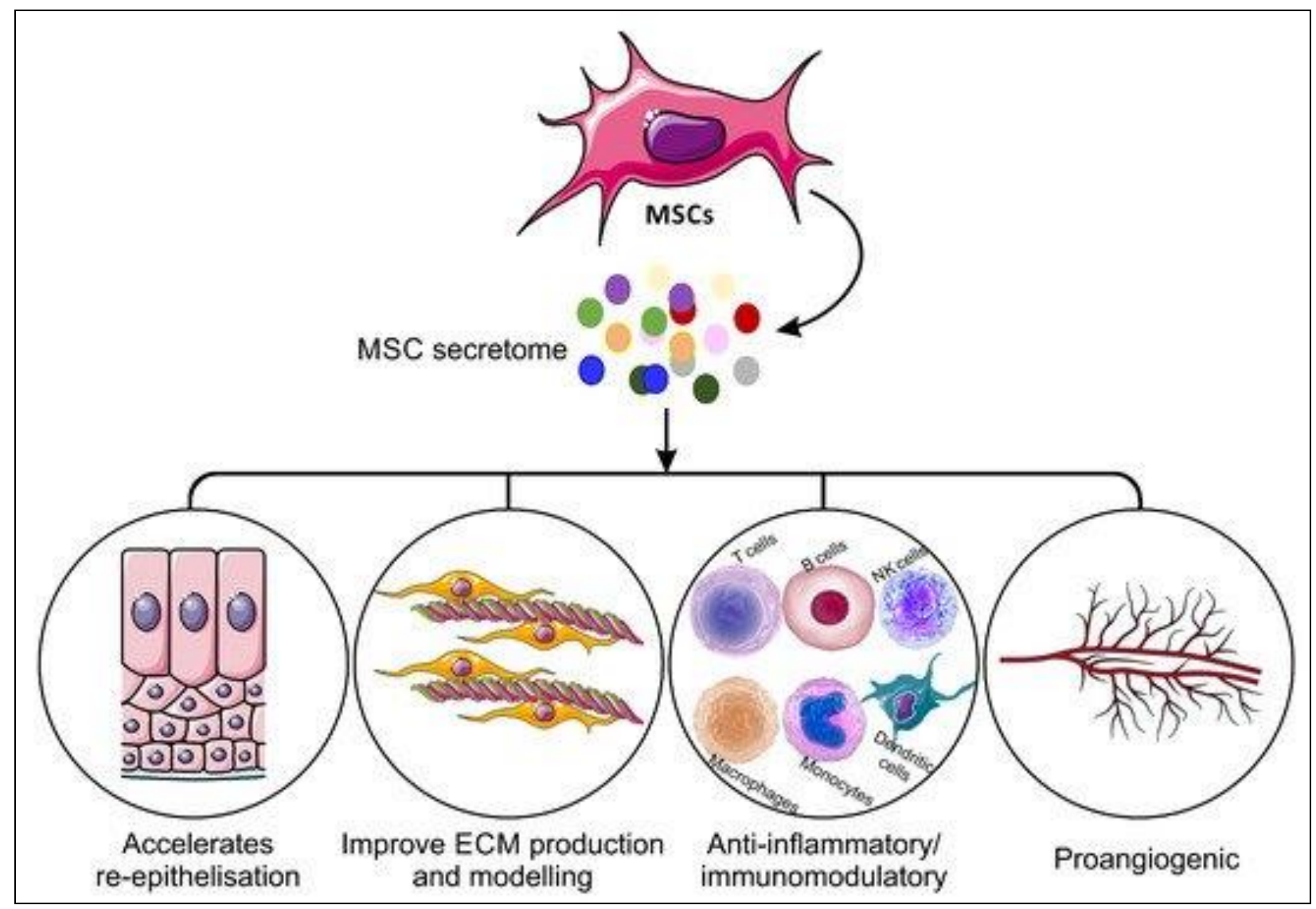

Fig 8: The effect of secretome produced by MSC in wound healing process. ${ }^{[13]}$

The secretome is present in the medium used to culture the stem cells; this medium is referred to as conditioned medium (CM). Secretome has several advantages over stem cells in terms of processing, storing, packaging, and transportation. Numerous studies have shown that MSC-CM contains growth factors that aid in the regeneration of damaged organ tissues, with a particular emphasis on proliferation, most notably epidermal growth factor (EGF), basic fibroblast growth factor (bFGF), and hepatocyte growth factor (HGF). It has been demonstrated that it accelerates wound healing by increasing the proliferation and migration of preexisting skin cells through PI3K / Akt or FAK-ERK1/2 signaling. In addition, proangiogenic proteins that contribute to angiogenesis are also found, such as Vascular Endothelial Growth Factor (VEGF), PlateletDerived Growth Factor (PDGF), Angiopoietin-1 (Ang-1), Ang-2, angiostatin, CXCL16, granulocyte-macrophage colony-stimulating factor (GM-CSF), monocyte chemotactic protein-1 (MCP-1), matrix metalloproteinase (MMP)-8 and MMP-9. Besides that, the secretome contains antiinflammatory cytokines (Transforming Growth Factor-Beta 1(TGF- 1 1), Interleukin-6 (IL-6), IL-10, IL-27, IL-17E, IL13 , and IL-12p70, as well as pro-inflammatory cytokines (IL-1 $\beta$, IL-8 / CXCL-8, and IL-9) that also make a significant contribution to wound healing ${ }^{[6,13,15]}$.

Antimicrobial effect can be achieved by secreting antimicrobial peptides (AMPs) and expressing molecules such as 3-dioxygenase (IDO), indoleamine-2, and interleukin (IL)-17. MSC have been shown in several studies to be capable of directly killing certain bacteria, including Pseudomonas aeruginosa, Klebsiella pneumoniae, Staphylococcus aureus, and Escherichia coli. By interfering with the integrity of the bacterial cell membrane or by enhancing chemotaxis and chemokine induction, the mechanisms are outlined below. Cathelicidin LL-37, human-defensin 2 (hBD-2), hepcidin, and lipocalin 2 are all AMPs expressed by human MSCs (Lcn2). AMP is one of the critical components produced by MSCs that aid in the control or elimination of bacterial infection and the regulation of inflammatory responses ${ }^{[16-18]}$.

Wharton's Jelly-derived MSCs (Wj-MSCs), one of the MSC sources derived from the umbilical cord, are considered to be more beneficial than adult mesenchymal stem cells. Without using an invasive method or additional surgery, $\mathrm{Wj-MSCs}$ are isolated from extraembryonic tissues discarded following delivery. Due to their nontumorigenicity and immunomodulatory properties, they can be allotransplanted without rejection to regenerate organs such as the liver, bone, fat, heart, cartilage, neural, pancreas, blood vessels, and skin components ${ }^{[11,19]}$.

$\mathrm{Wj}$-MSCs release anti-inflammatory, proangiogenic, and other molecules that can promote wound healing, such as IL-10, TGF- $\beta$, Prostaglandin $\mathrm{E}_{2}$ (PGE2), IDO, and TNF $\alpha-$ stimulated gene-6 (TSG-6), VEGF, bFGF, IL-6, PDGF, Placental Growth Factor (PlGF), Ang-1, MCP-1, and HGF $[11,20]$.

According to several studies, Wj-MSCs increase reepithelialization, fibroblast proliferation, neovascularization, and collagen deposition, all of which can accelerate wound closure. Wj-MSCs can be used as an alternative therapy option in wound healing to stimulate wound repair ${ }^{[11,21]}$.

\section{Conclusion}

Echtyma gangrenosum is a skin disease that begins as an erythematous macule and rapidly progresses to a gangrenous ulcer with a black eschar surrounded by 
erythema. Due to its proliferative and regenerative potentials, as well as its strong antimicrobial effect, Secretom of Warthon Jelly Mesechymal Stem cell therapy may be an alternative therapy for EG. A case of EG in a previously healthy woman treated with intracutaneous injection and topical gel of Wharton's Jelly-derived MSCs (Wj-MSCs) secretome demonstrated a significant improvement in the wound healing process, and the patient was satisfied with the results.

\section{References}

1. Zhu CY, Zhang GX, Yu ZZ, Li ZJ, Fan YM. Pseudomonas aeruginosa ecthyma gangrenosum in a woman with recurrent Graves' disease. Int. J Infect. Dis 2014;21:19-20.

2. Lian F. Clinicopathologic Aspects of Ecthyma Gangrenosum in Pediatric Patients a Case Series and Review of the Literature. J Clin. Anat. Pathol 2013;1:15.

3. Gençer S, Özer S, Ege Gül A, Doğan M, Ak Ö. Ecthyma gangrenosum without bacteremia in a previously healthy man: A case report. J Med. Case Rep 2008;2:1-3.

4. Sukmawati Tansil Tan, Yohanes Firmansyah, Jessica Elizabeth. New approach to skin burn treatment: Potential of secretome from Wharton's jelly mesenchymal stem cell therapy. Int $\mathrm{J}$ Adv Biochem Res. 2020;4(2):11-16. DOI: $10.33545 / 26174693.2020 . v 4.12 a .49$

5. Martínez-Longoria CA, Ocampo-Garza J, OcampoCandiani J, Rosales-Solis GM, Guerrero-González GA. Ecthyma gangrenosum: A report of eight cases. An. Bras. Dermatology. 2017;92:698-700.

6. Julianto I, Rindastuti Y. Topical Delivery of Mesenchymal Stem Cells "Secretomes" in Wound Repair 217-220.

7. Biscaye $\mathrm{S}$, et al. Ecthyma gangrenosum, a skin manifestation of Pseudomonas aeruginosa sepsis in a previously healthy child: A case report. Med. (United States) 2017;96:2-5.

8. Funk E, Ivan D, Gillenwater AM. Ecthyma Gangrenosum. Arch Otolaryngol Head Neck Surg 2009;135:818-820.

9. Singh TN, Devi KM, Devi KS. Ecthyma gangrenosum: A rare cutaneous manifestation caused by Pseudomonas aeruginosa without bacteraemia in a leukaemic patienta case report. Indian J Med. Microbiol 2005;23:262263.

10. Kirby GTS, Mills SJ, Cowin AJ, Smith LE. Stem cells for cutaneous wound healing. Biomed Res. Int 2015.

11. García-Guillén AI. Wharton'S Jelly Mesenchymal Stem Cell Therapy For Skin Wound Healing. J Stem Cells Res. Dev. Ther. 2020;6:1-6.

12. Samakova A et al. The pi3k/Akt pathway is associated with angiogenesis, oxidative stress and survival of mesenchymal stem cells in pathophysiologic condition in ischemia. Physiol. Res 2019;68:S131-S138.

13. Ahangar P, Mills SJ, Cowin AJ. Mesenchymal stem cell secretome as an emerging cell-free alternative for improving wound repair. Int. J. Mol. Sci 2020;21:1-15.

14. Lee DE, Ayoub N, Agrawal DK. Mesenchymal stem cells and cutaneous wound healing: Novel methods to increase cell delivery and therapeutic efficacy. Stem Cell Res. Ther 2016;7:1-8.
15. Park S et al. Stem Cell Secretome and Its Effect on Cellular Mechanisms Relevant to Wound Healing. Mol. Ther 2018;26:606-617.

16. Alcayaga-Miranda F, Cuenca J, Khoury M. Antimicrobial activity of mesenchymal stem cells: Current status and new perspectives of antimicrobial peptide-based therapies. Front. Immunol 2017, 8.

17. Chow $\mathrm{L}$ et al. Antibacterial activity of human mesenchymal stem cells mediated directly by constitutively secreted factors and indirectly by activation of innate immune effector cells. Stem Cells Transl. Med 2020;9:235-249.

18. Johnson V et al. Activated Mesenchymal Stem Cells Interact with Antibiotics and Host Innate Immune Responses to Control Chronic Bacterial Infections. Sci. Rep 2017;7:1-18.

19. AI A et al. Human Wharton's jelly mesenchymal stem cells promote skin wound healing through paracrine signaling. Stem Cell Res. Ther 2014;5:1-13.

20. Tan ST, Firmansyah Y, Elizabeth J. New approach to deep diabetic foot ulcer (DFU) treatment-potential of secretome from Wharton's jelly mesenchymal stem cell therapy. Int. J. Dermatology, Venereol. Lepr. Sci 2020;3:21-26.

21. Zhao G et al. Large-scale expansion of Wharton's jellyderived mesenchymal stem cells on gelatin microbeads, with retention of self-renewal and multipotency characteristics and the capacity for enhancing skin wound healing. Stem Cell Res. Ther 2015;6:1-16.

22. Varghese G, Eapen P, Abraham S. Ecthyma gangrenosum of a single limb. Indian J. Crit. Care Med 2011;15:188-189. 